Psychiatry

Elsevier Editorial system(tm) for European

Manuscript Draft

Manuscript Number: EURPSY-D-17-00068R1

Title: How is psychotherapy training perceived by psychiatric trainees? A cross-sectional observational study in Europe

Article Type: Viewpoint

Keywords: Education in psychiatry; Psychiatry in Europe; Psychotherapy

Corresponding Author: Mr. Thomas Gargot,

Corresponding Author's Institution: Hopital de la Pitie Salpetriere, Institut des systèmes inteligents et de la robotique, Université Pierre et Marie Curie, Paris, France

First Author: Thomas Gargot

Order of Authors: Thomas Gargot; Clément Dondé; Nikitas Arnaoutoglou; Roberts Klotins; Petra Marinova; Rita Silva; Ekin Sönmez

Manuscript Region of Origin: FRANCE

Abstract: Background: Current recommendations on psychotherapy training for psychiatric trainees in Europe are available from the European Federation of Psychiatric Trainees (EFPT) and the European Union of Medical Specialists (UEMS). Despite trainees' high motivation and the acknowledged effectiveness of psychotherapy, recent studies have shown a significant lack of training. No study so far has focused on a large European sample.

Methods: From 2013 to 2015, the EFPT conducted an online cross-sectional survey among psychiatric trainees and early career psychiatrists from 22 European countries.

Results: Out of 572 respondents, 92\% considered psychotherapy important for their professional identity, 90\% wanted to practice psychotherapy after their training. Respondents were interested in a great variety of psychotherapies. Almost half underwent psychotherapy training, a third did not have any training experience in psychotherapy. Less than one half had access to supervision. Only 9\% were satisfied with the level of funding of their psychotherapy training. Although recommendations of the EFPT and UEMS are widely recognised, they are not implemented consistently.

Conclusions: There is a large mismatch between the high motivation of respondents to train in psychotherapy and the actual training opportunities available. The EFPT strongly supports initiatives that can improve psychotherapy training. We believe that wider availability of high-quality online learning resources for trainees, as well as international collaborations between psychiatry training associations and psychotherapy associations could be a step in the right direction to bridge this gap. 


\title{
How is psychotherapy training perceived by psychiatric trainees? A cross-sectional
}

\author{
observational study in Europe
}

\section{Background}

Evidence for efficacy of psychological therapies is growing and the inclusion of such therapies in international guidelines for treatment of psychiatric disorders is increasing as well. Accessibility of psychotherapeutic treatment for the patients can be improved through providing improvements in training of psychotherapists - including psychiatry trainees. European organisations have published guidelines requiring programs to promote psychotherapeutic competences among psychiatry trainees, for example the European Psychiatric Association [1,2] and the EFPT (European Federation of Psychiatric trainees) [3], whose statements are largely concordant with the UEMS (Union Européenne des Médecins Spécialistes) statements [4]. However, the implementation of these recommendations and the quality of psychotherapy training appears to be poor among European countries $[5,6]$. We aimed to assess the availability of psychotherapy training for psychiatric trainees in Europe and trainee level of satisfaction. Furthermore, we assessed the awareness and implementation of the UEMS recommendations.

\section{Methods}

We carried out a cross-sectional survey from January 2013 to October 2015. An online questionnaire was designed by the EFPT Psychotherapy Working Group, as a result of collective debates considering previous studies [5,6] and psychotherapy training-related European recommendations [2-4]. The target population was meant to be psychiatrists in training or those within 5 years from qualification. We established national coordinators for each country to spread the questionnaire via local trainee networks, In some large countries there was no way for them to estimate accurately how many trainees there were but we estimate that there might be 20000 psychiatric trainees in Europe. Two reminders were sent out across the survey period for each participating country. The questionnaire was implemented using Open Source software - Limesurvey. Further technical details as well as the data and analysis files are available at http://github.com/EFPT/efptPWG. 


\section{Results}

Five hundred and seventy-four (574) respondents returned the survey, with a response rate of $2.9 \%$ of estimated number of European psychiatry trainees. We collected answers from 22 European countries: Romania $(n=93)$, France $(n=64)$, Slovenia $(n=60)$, Czech Republic $(n=58)$, Italy $(n=53)$, Greece $(n=43)$, Israel $(n=35)$, Belgium $(n=26)$, Spain $(n=24)$, Germany $(n=24)$, Finland $(n=24)$, Bulgaria $(n=19)$, Latvia $(n=10)$, Bosnia and Herzegovina $(n=10)$, Lithuania ( $n=7)$, Estonia $(n=7)$, Albania $(n=7)$, Malta $(n=5)$, Switzerland $(n=2)$, UnitedKingdom ( $\mathrm{n}=1)$, Croatia $(\mathrm{n}=1)$, Belarus $(\mathrm{n}=1)$.

They show very significant interest and motivation for psychotherapy but available resources appear scarce. Recommendations of UEMS are reported to be known by $51 \%$ of respondents and implemented in $22 \%$ of respondents' country of origin. Data analyses for the whole sample are provided Table 1.

The respondents from two countries (Croatia and Belarus, $n=3$ ) were excluded from the comparisons between countries because there was not at least one answer by variable of interest. The only respondent from United-Kingdom was also excluded. With exception from Malta, Germany and Israel, less than $50 \%$ of respondents had received any training in psychotherapy within their psychiatry program. On average 1 out of 5 trainees reported to have received psychotherapy, but such an effort or a more comprehensive training were undertaken on one's own initiative for $40 \%$ of respondents, and more than $50 \%$ of those from Lithuania, Bulgaria, Greece, Spain and Czech Republic paid for this experience from their own pocket. Ninety percent $(90 \%)$ of respondents wished to practice psychotherapy in the future. Trainees from 8 out of 20 countries gave a mean "satisfied" rate concerning PT opportunities and components in their psychiatric curricula. Trainees from Malta, Belgium, Israel and Germany were the most satisfied. Trainees from Albania, Latvia, Bosnia and 
Herzegovina were the least satisfied. Only one country (Malta) gave a mean "satisfied" rate concerning psychotherapy training funding within their psychiatric curricula. All other respondents were, on average, dissatisfied concerning this aspect, especially those from Albania, Slovenia, and Bulgaria. Mean duration of supervision per month was highest in Bosnia, Israel, Malta and Switzerland with more than 8 hours. Lowest availability of psychotherapy related supervision was found to be in Albania, Belgium, Finland, Estonia and Czech Republic with less than 3 hours per month.

\section{Discussion}

To our knowledge this is the first large survey focusing on the topic of psychotherapy component in psychiatric training in Europe.

The results reveal a large mismatch between the high motivation of trainees who want to be trained in psychotherapy, and the relatively limited resources available for such training. We observe low rates of satisfaction (35\%) with components of psychotherapy training, similar as in a previous study [7], which is likely associated with poor availability of supervision and public funding for psychotherapy training for psychiatrists. By contrast, respondents show strong interest in psychotherapy, as $90 \%$ of them wanted to be able to practice psychotherapy after finishing their psychiatry training, which is consistent with a recent world survey in which $80 \%$ of interviewed early career psychiatrists trusted psychotherapy as a valid method of treatment for psychiatric disorders [7]. Financial burdens of psychotherapy training were highlighted in the World Psychiatry Association study [7]. Our work not only confirms this burden but also shows high motivation of trainees through the fact that $96 \%$ of respondents would invest their time to train in psychotherapy if they did not have to pay for training out of their own pocket. Furthermore trainees said they would be prepared to pay on average $9 \%$ of their yearly salary in order to learn psychotherapy. 
We find that UEMS recommendations for psychotherapy training component in psychiatry training are well known but poorly implemented. Comparing the awareness of UEMS recommendations for psychotherapy training in psychiatry with the trainee perception of their actual implementation shows a large mismatch. UEMS points out that psychotherapy training should be publicly funded, whereas the majority of respondents have to pay for each PT component.

In a previous survey conducted among European psychiatry trainees, Nawka et al. have also pointed out similar challenges, namely the shortfall of patients and supervision made available by psychiatric centres for psychotherapy practice opportunities and lack of financial resources [8]. Lectures are the only mandatory psychotherapy training component in the curriculum for a large part of our respondents. Personal psychotherapy experience was undertaken by a minority of respondents, however both UEMS and EFPT statements recommend such experience. The low implementation of recommendations might reflect the poor communication from the academic societies and associations to the training centres. To assess this point properly, we think, trainers should also be asked about their awareness of psychotherapy training recommendations and their implementation.

We observed differences between countries concerning important components of psychotherapy training. Inequalities in psychotherapy training distribution may be viewed as an indirect result of uneven distribution of mental health and general health services among European countries, but more comprehensive data would be needed for further analysis.

Given the findings of our survey, our interpretation for the best way to incorporate psychotherapy skills into trainee daily medical practice would be implementation of evidencebased psychotherapy training courses in psychiatric trainee curricula, that should reflect trainee interest in several methods of treatment. Actual UEMS and EFPT recommendations 
are meant to address this challenge. The main issue in implementing the recommendations might be the communication between the national psychiatric associations and training centres. A European network of trainees, such as EFPT, could help gather updated resources and disseminate them to improve the quality of psychotherapy training (e.g. http://efpt.eu/usefull-links/). European psychiatric and psychotherapy associations could play a major role in bridging gaps between the reality of psychotherapy training and the trainees' demand, by pursuing high-quality psychotherapy courses arrangements. This should further improve the future scope and quality of care provided for psychiatric patients as psychiatrists are an influential part in mental health care systems.

This survey suffers from the common limitation of generalizability. It was not possible for us to conclude that we have reached a representative sample of psychiatric trainees, as the study sample size was relatively low and does not represent all Europe. Variations in the number of responses from individual countries impair the generalizability of the results. Trainees with an inclination towards psychotherapy could have been more disposed to respond and support improvement for psychotherapy training. Since the respondents were recruited through EFPT links to national trainees' associations, it is possible our respondents were more aware of the related guidelines.

\section{Conclusions}

This survey shows the need for improved psychotherapy training for European psychiatrists. The trainee motivation for psychotherapy training and awareness of European recommendations were high. However, trainees from their own experience thought their psychotherapy training and respective implementation of UEMS recommendations for psychotherapy training were insufficient. European psychiatric and psychotherapy associations could have a major role in making high-quality psychotherapy training more 
readily available for the future psychiatrists and therefore improve the future care of psychiatry patients in national mental health care systems.

\section{Conflicts of interest and funding sources}

All authors state that they have no conflict of interest or funding sources related to this study.

\section{Acknowledgements}

We are grateful to Prof. Klaus Ebmeier who provided his valuable input and comments.

\section{References}

[1] Bhugra D, Ventriglio A, Kuzman MR, Ikkos G, Hermans MH-M, Falkai P, et al. EPA guidance on the role and responsibilities of psychiatrists. Eur Psy 2015;(30):417-22.

[2] Mayer S, van der Gaag RJ, Dom G, Wassermann D, Gaebel W, Falkai P, et al. European Psychiatric Association (EPA) guidance on post-graduate psychiatric training in Europe. Eur Psy 2014;29(2):101-6.

[3] EFPT Statements, http://efpt.eu/wp-content/uploads/2014/07/STATEMENTS-OFTHE-EUROPEAN-FEDERATION-OF-PSYCHIATRIC-TRAINEES-1.docx; 2016 [accessed 25.01.17].

[4] UEMS Board of Psychiatry, Charter on training of medical specialists in the EU, http://uemspsychiatry.org/wp-content/uploads/2013/09/Chapter6-11.10.03.pdf; 2013 [accessed 25.01.17].

[5] Fiorillo A, Luciano M, Giacco D, Del Vecchio V, Baldass N, De Vriendt N. Training and practice of psychotherapy in Europe: results of a survey. World Psychiatry. 2011;(3):238.

[6] van Effenterre A, Hanon C, Llorca P-M. Enquête auprès des PU-PH sur la formation en psychiatrie en France. L'Encéphale 2014;(40):208-15.

[7] Nawka, A, Kuzman MR, Giacco D, Malik A. Challenges of Postgraduate Psychiatric Training in Europe: A Trainee Perspective. Psyc Services 2010;61(9):862-64.

[8] Carmel A, Shapiro DI. Development of an Evidence-Based Psychotherapy Seminar for Psychiatry Residency Training. Acad Psychiatry 2016;(40):188-9. 
[9] Weerasekara P, Manring J, Lynn D. Psychotherapy training for residents: reconciling requirements with evidence-based, competence focused practice. Acad Psychiatry 2010(34):5-12. 


\section{Socio-demographics}

\section{Number of respondents}

Responding countries

Sex ratio

Mean age

Status

\section{Interest in psychotherapy}

Consider psychotherapy as important

for their professional identity

Want to practice psychotherapy after psychiatry training

Psychotherapy type interest

Involvement if possibility of free $P T$

Involvement if possibility of paying $P T$

\section{2 / 20000 (response rate $=2.9 \%)$}

22

$69 \%$ female $(n=395), 31 \%$ male $(n=177)$

32 years old (sd: 5.3 )

$80 \%$ trainees $(n=479), 19 \%$ early career $(n=93)$

\section{Current situation in PT}

Training in $P T$

Not involved in a $P T$

Completed a full $P T$

Undergone personal psychotherapy

Undertook PT on their own initiative

Qualified for psychotherapy at the end

of psychiatry training

Beneficing from supervision

Mean hours of supervision per month

$92 \%$ [95\% Cl: 0.89-0.94] $(n=508)$

$90 \%$ [95\% Cl: 0.87-0.92] $(n=495)$

$60 \%$ psychodynamic $(n=345), 46 \%$ CBT $(n=266)$,

$26 \%$ systemic $(n=150), 1 \%$ group

$(n=9), 28 \%$ other $(n=165), 32 \%$ more than one type simultaneously $(n=222)$

$96 \%$ [95\% Cl: 0.93-0.97] ( $\mathrm{n}=461)$

$60 \%(n=279)$ [95\% Cl: $0.56-0.65]$ would give $>5 \%$ of their salary for PT

\section{Compulsory in psychiatry training}

Theoretical lectures

Practice of psychotherapy

Personal psychotherapy

$48 \%$ [95\% Cl: $0.44-0.52](n=329)$

$32 \%$ [95\% Cl: $0.28-0.35](n=213)$

$5 \% \quad[95 \% \mathrm{Cl}: 0.04-0.07](\mathrm{n}=36)$

$40 \%$ [95\% Cl: $0.37-0.45](n=234)$

$33 \%$ [95\% Cl: $0.30-0.37](n=229)$

$38 \%$ [95\% Cl: 0.34-0.42] $(n=193)$

$38 \%$ [95\% Cl: $0.33-0.42](n=187)$

1.36 hours [95\% Cl: $1.16-1.51]$

$70 \%$ [95\% Cl: $0.66-0.74](n=362)$

$43 \%$ [95\% Cl: $0.38-0.47](n=219)$

$22 \%$ [95\% Cl: $0.19-0.26](n=113)$

\section{Paid by state (publicly funded)}

Theoretical lectures

$36 \%$ [95\% Cl: 0.31-0.40] $(\mathrm{n}=171)$

$26 \%$ [95\% Cl: 0.22-0.30] $(n=125)$

\section{Satisfaction with current PT}

Satisfied with PT delivered in their country

Satisfied with psychotherapy components

of their psychiatry training

Satisfied with funding provided for $P T$ 


\section{UEMS recommendations for PT}

Total

$>100$ hours of supervision

$>120$ hours of theory

Supervisors should be qualified

Personal therapy experience

$P T$ as a mandatory part of the

psychiatric curriculum

Defined number of cases should be seen

Work experience with individuals,

families and groups

Training to various type of psychotherapy

PT should be publicly founded

\section{Awareness}

$51 \%$

$45 \%$

$40 \%$

$75 \%$

$58 \%$

$55 \%$

$51 \%$

$48 \%$

$45 \%$
$41 \%$

\section{Implementation in country}

$22 \%$

$30 \%$

$25 \%$

$53 \%$

$41 \%$

$36 \%$

$33 \%$

$30 \%$

$25 \%$

$25 \%$

Table 1: Data analyses of the cross-sectional survey, whole european sample. PT = psychotherapy training. 
Title:

How is psychotherapy training perceived by psychiatric trainees? A cross-sectional observational study in Europe

\section{Authors:}

Thomas Gargot ${ }^{1}$, Clément Dondé ${ }^{2}$, Nikitas Arnaoutoglou ${ }^{3}$, Roberts Klotins ${ }^{4}$, Petra Marinova ${ }^{5}$, Rita Silva ${ }^{6}$, Ekin Sönmez ${ }^{7}$, EFPT Psychotherapy Working Group ${ }^{8}$

\section{Affiliations:}

${ }^{1}$ Département de psychiatrie de l'enfant et de l'adolescent, Hôpital de la Pitié Salpêtrière, Institut des systèmes intelligents et de la Robotique, Paris, France, thomas_gargot@hotmail.com

${ }^{2}$ INSERM U1028, CNRS UMR5292, PSYR2 Team, Lyon Neuroscience Research Center, Centre Hospitalier Le Vinatier, Bron, Université Claude Bernard Lyon 1, Lyon, France, clement.dondecoquelet@,gmail.com

${ }^{3}$ Department of Psychiatry, University of Oxford, Oxford, UK, nikitas.arnaoutoglou@psych.ox.ac.uk

${ }^{4}$ Tavistock and Portman NHS Foundation Trust, Adult Department, 120 Belsize Lane, London, NW3 5BA, UK, roberts.klotins@gmail.com

${ }^{5}$ Private psychiatric practice, Sofia, Bulgaria, petra.marinova@gmail.com

${ }^{6}$ Department of Psychiatry and Mental Health of the Baixo Vouga Hospital Centre, Portugal, rita.mcrs@gmail.com

${ }^{7}$ Marmara University School of Medicine, Department of Psychiatry, Istanbul, Turkey, ekinsonmez88@gmail.com

${ }^{8}$ EFPT Psychotherapy Working Group 2016-2017, http://efpt.eu/wg/psychotherapy-wg/, psychotherapy-wg@efpt.eu

\section{Person handling the correspondence at all stages of refereeing, publication and post- publication:}

Thomas Gargot

Département de psychiatrie de l'enfant et de l'adolescent, Hôpital de la Pitié Salpêtrière, 4783, boulevard de l'Hôpital 75651 PARIS Cedex 13, France, thomas_gargot@hotmail.com 


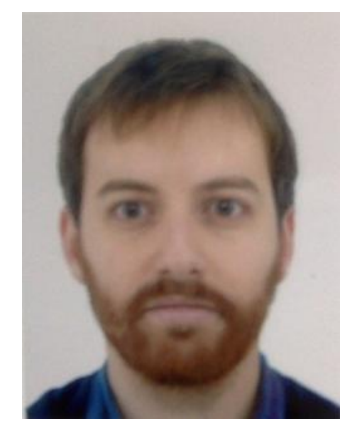

Thomas GARGOT is a trainee in psychiatry in Paris, France in La Pitié Salpêtrière Hospital. He is also the chair of the EFPT psychotherapy working group and EFPT Information and Technologies secretary. He collects online websites and European events that could help psychiatry trainees http://efpt.eu/usefull-links/forum-school-moocs/. He is interested in cognitive and behavioral therapies especially motivational interviewing and psychoeducation. He did a master degree in cognitive sciences about automatic extraction of social signal on psychotherapies videos (https://github.com/Ouphix/synchro-psychotherapies/). He is interested in open science tools to improve research in psychiatry. 


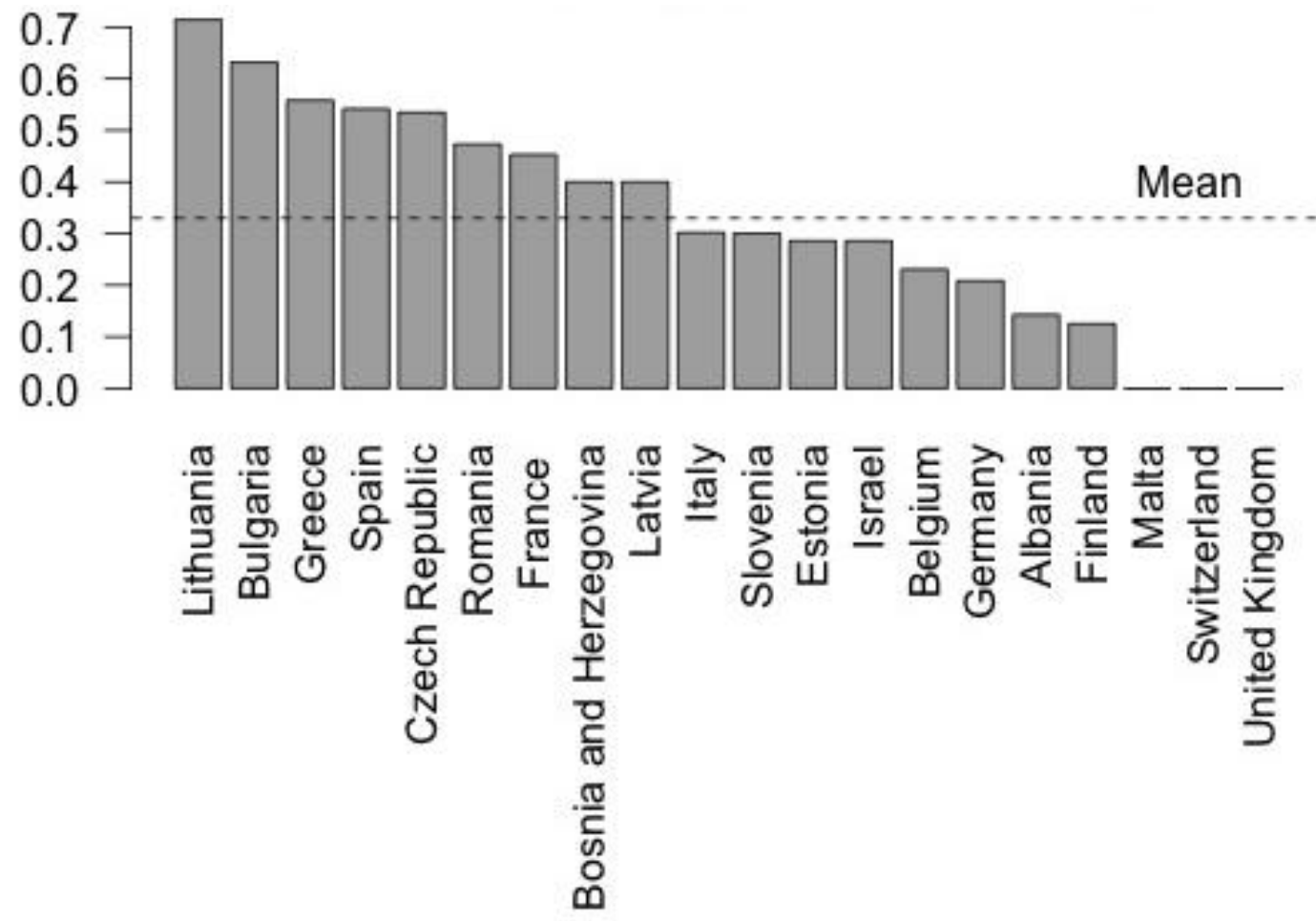

Figure S1: Country percentages of respondents undertaking psychotherapy training on own initiative. 


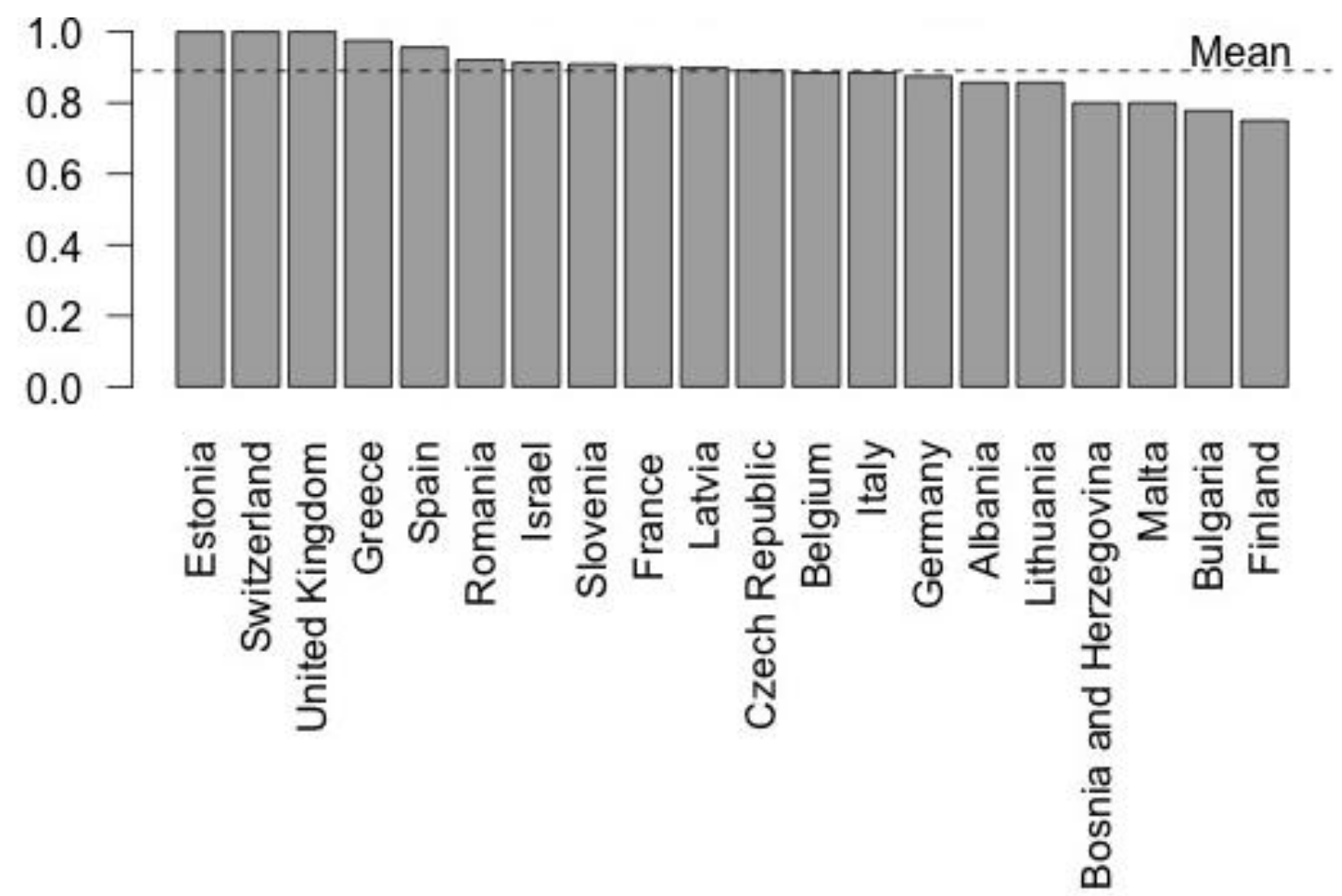

Figure S2: Country percentages of respondents who would like to practice psychotherapy training in the future. 

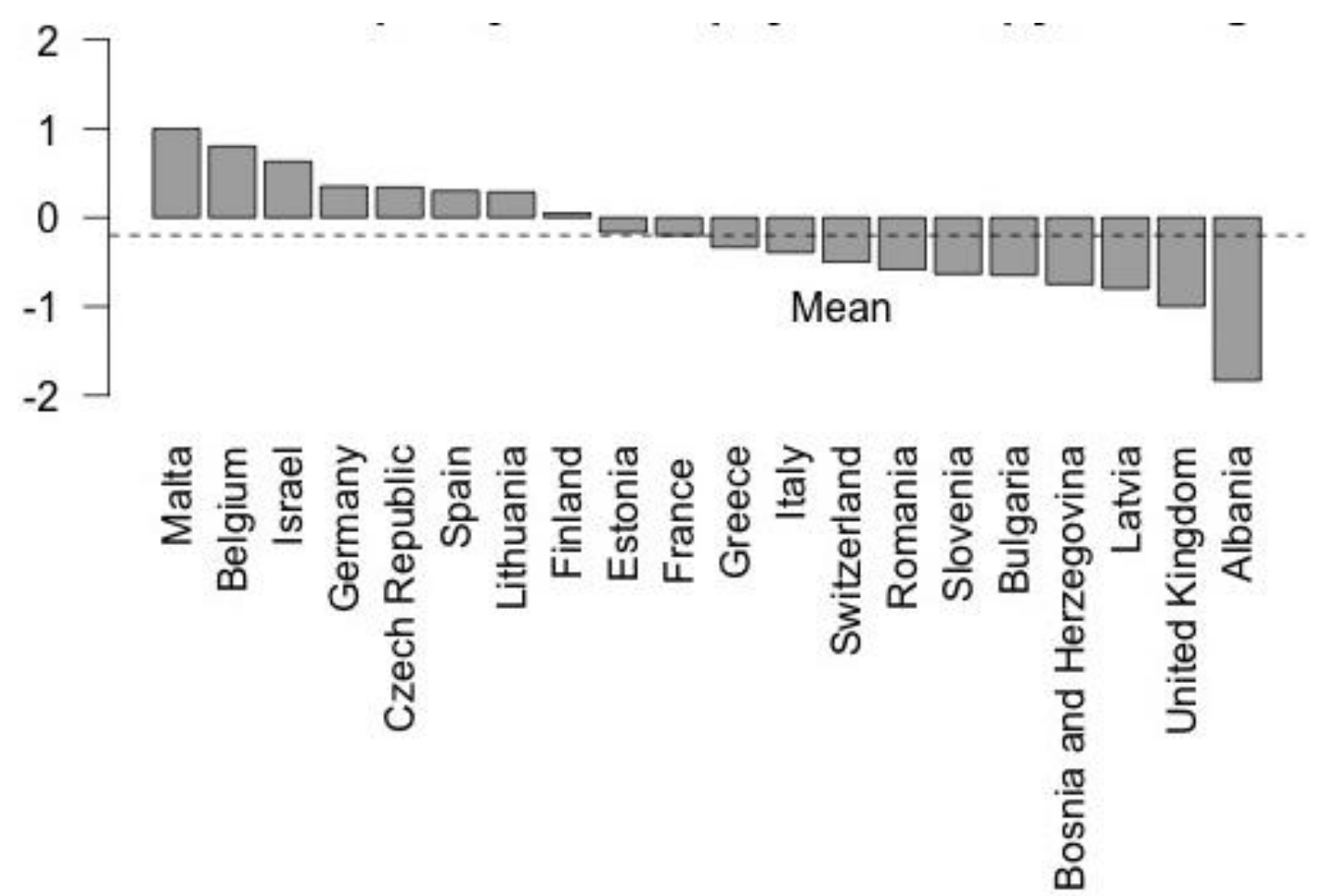

Figure S3: Country mean satisfaction of respondents regarding the quality of their psychotherapy training components within the psychiatric curricula. $2=$ very satisfied, $1=$ satisfied, $0=$ Neutral, $-1=$ dissatisfied, $-2=$ very dissatisfied. 


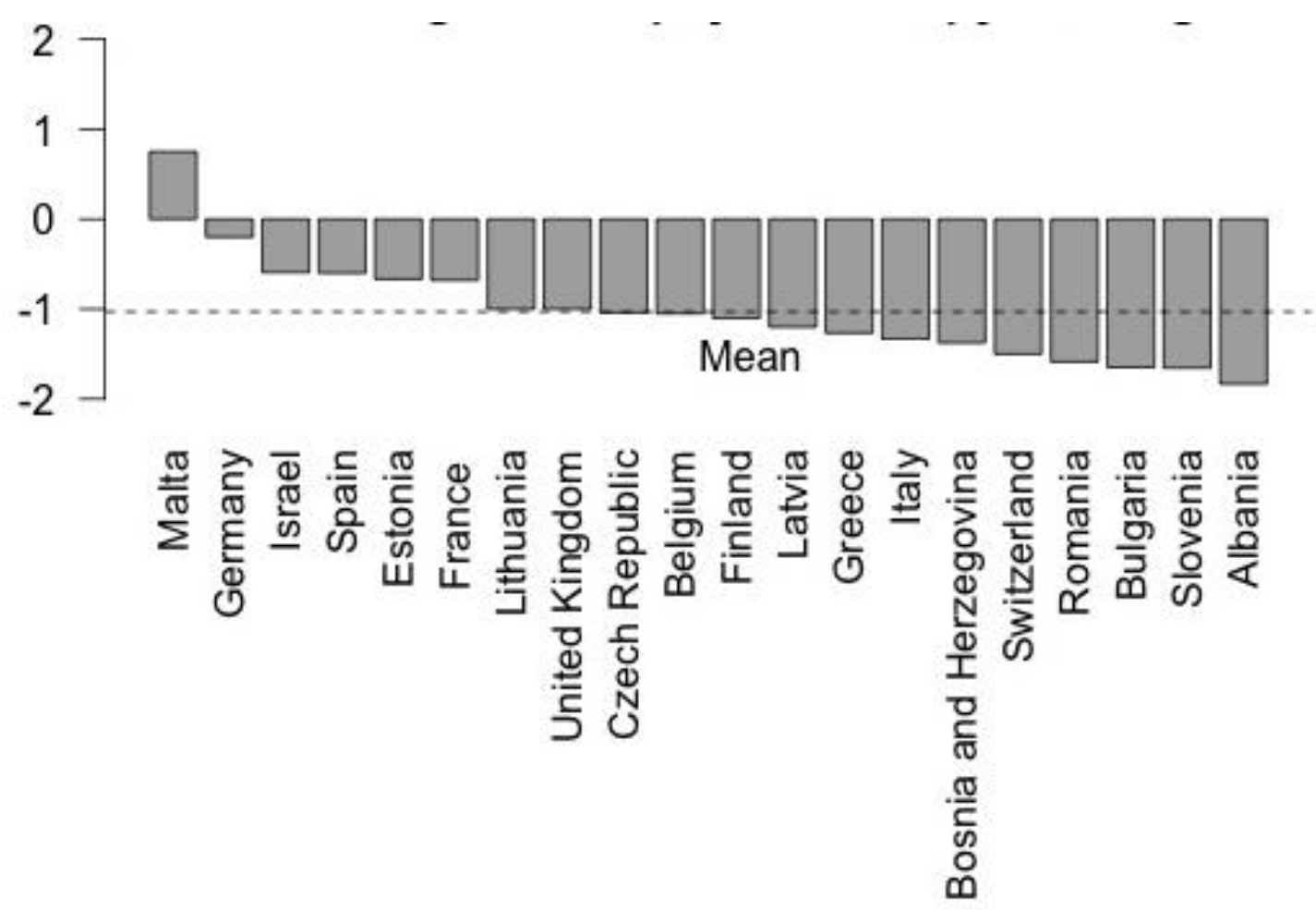

Figure S4: Country mean satisfaction of respondents regarding the funding of their psychotherapy training within the psychiatric curricula. $2=$ very satisfied, $1=$ satisfied, $0=$ Neutral, -1 = dissatisfied, $-2=$ very dissatisfied. 